\title{
Constraining bright optical counterparts of fast radio bursts
}

\author{
Consuelo Núñez ${ }^{1}$, Nicolas Tejos ${ }^{1}$, Giuliano Pignata ${ }^{2,3}$, Charles D. Kilpatrick ${ }^{4}$, J. Xavier Prochaska ${ }^{5,6}$, \\ Kasper E. Heintz ${ }^{7,8,9}$, Keith W. Bannister ${ }^{10}$, S. Bhandari ${ }^{10}$, Cherie K. Day ${ }^{11,10}$, A. T. Deller ${ }^{11}$, Chris Flynn ${ }^{11,12}$, \\ Elizabeth K. Mahony ${ }^{10}$, Diego Majewski ${ }^{5}$, Lachlan Marnoch ${ }^{13,10,14}$, Hao Qiu ${ }^{15}$, Stuart D. Ryder ${ }^{13}$, ${ }^{4}$, \\ Ryan M. Shannon ${ }^{11}$ \\ 1 Instituto de Física, Pontificia Universidad Católica de Valparaíso, Casilla 4059, Valparaíso, Chile \\ e-mail: consuelo.nunez.p@mail.pucv.cl; nicolas.tejos@pucv.cl \\ 2 Departamento de Ciencias Físicas, Universidad Andres Bello, Avda. Republica 252, Santiago, Chile \\ ${ }^{3}$ Millennium Institute of Astrophysics, Nuncio Monseñor Sótero Sanz 100, Providencia, Santiago, Chile \\ ${ }^{4}$ Center for Interdisciplinary Exploration and Research in Astrophysics (CIERA) and Department of Physics and Astronomy, North- \\ western University, Evanston, IL 60208, USA \\ ${ }^{5}$ University of California Observatories-Lick Observatory, University of California, 1156 High Street, Santa Cruz, CA95064, USA \\ ${ }^{6}$ Kavli Institute for the Physics and Mathematics of the Universe (WIP), 5-1-5 Kashiwanoha, Kashiwa, 277-8583, Japan \\ 7 Centre for Astrophysics and Cosmology, Science Institute, University of Iceland, Dunhagi 5, 107 Reykjavík, Iceland \\ 8 Cosmic Dawn Center (DAWN), Denmark, \\ 9 Niels Bohr Institute, University of Copenhagen, Jagtvej 128, 2200 Copenhagen N, Denmark \\ 10 Australia Telescope National Facility, CSIRO Astronomy and Space Science, PO Box 76, Epping, NSW 1710, Australia \\ 11 Centre for Astrophysics and Supercomputing, Swinburne University of Technology, Hawthorn, VIC 3122, Australia \\ 12 ARC Centre of Excellence for Gravitational Wave Discovery (OzGrav), Australia \\ 13 Department of Physics and Astronomy, Macquarie University, NSW 2109, Australia \\ 14 Astronomy, Astrophysics and Astrophotonics Research Centre, Macquarie University, Sydney, NSW 2109, Australia \\ 15 Sydney Institute for Astronomy, School of Physics, University of Sydney, NSW 2006, Australia
}

July 23, 2021

\begin{abstract}
Context. Fast radio bursts (FRBs) are extremely energetic pulses of millisecond duration and unknown origin. To understand the phenomenon that emits these pulses, targeted and un-targeted searches have been performed for multiwavelength counterparts, including the optical.

Aims. The objective of this work is to search for optical transients at the positions of eight well-localized $\left(<1^{\prime \prime}\right)$ FRBs after the arrival of the burst on different timescales (typically at one day, several months, and one year after FRB detection). We then compare this with known optical light curves to constrain progenitor models.

Methods. We used the Las Cumbres Observatory Global Telescope (LCOGT) network to promptly take images with its network of 23 telescopes working around the world. We used a template subtraction technique to analyze all the images collected at differing epochs. We have divided the difference images into two groups: In one group we use the image of the last epoch as a template, and in the other group we use the image of the first epoch as a template. We then searched for optical transients at the localizations of the FRBs in the template subtracted images.

Results. We have found no optical transients and have therefore set limiting magnitudes to the optical counterparts. Typical limits in apparent and absolute magnitudes for our LCOGT data are $~ 22$ and -19 mag in the $r$ band, respectively. We have compared our limiting magnitudes with light curves of super-luminous supernovae (SLSNe), Type Ia supernovae (SNe Ia), supernovae associated with gamma-ray bursts (GRB-SNe), a kilonova, and tidal disruption events (TDEs).

Conclusions. Assuming that the FRB emission coincides with the time of explosion of these transients, we rule out associations with SLSNe (at the $~ 99.9 \%$ confidence level) and the brightest subtypes of SNe Ia, GRB-SNe, and TDEs (at a similar confidence level). However, we cannot exclude scenarios where FRBs are directly associated with the faintest of these subtypes or with kilonovae.
\end{abstract}

Key words. fast radio burst - supernovae: general - techniques: photometric

\section{Introduction}

Fast radio bursts (FRBs) are extremely energetic radio frequency pulses that last for milliseconds or less (see, e.g., Cordes \& Chatterjee 2019, for a review). The dispersion measure (DM) of FRBs is greater than the expected contribution of the Milky Way (e.g.,, Petroff et al. 2016), which implies they are extragalactic. These signals come from all directions in the sky, and a sky rate of $[818 \pm 64 \text { (stat. })_{-200}^{+220}$ (sys.) $\mathrm{sky}^{-1} \mathrm{day}^{-1}$ above a fluence of
$5 \mathrm{Jy} \mathrm{ms}$ at $600 \mathrm{MHz}$ has been estimated (The CHIME/FRB Collaboration et al. 2021).

Hundreds of FRBs have been reported thus far by different radio telescopes (e.g., Lorimer et al. 2007; Spitler et al. 2014 Masui et al.|2015, Caleb et al. 2017, Shannon et al.|2018, Bhandari et al. 2018, Fedorova \& Rodin 2019; Ravi et al. 2019; Macquart et al. 2019; Law et al. 2020; Connor et al. 2020; The CHIME/FRB Collaboration et al.|2021), but only a dozen of them are localized to subarcsecond precision (Chatterjee et al. 2017, Bhandari et al. 2020; Marcote et al. 2020). For these 
Table 1. LCOGT observation summary.

\begin{tabular}{cccccc}
\hline \hline Field & $\begin{array}{c}\text { Date } \\
\text { (UTC) }\end{array}$ & Site $^{a}$ & $\begin{array}{c}\text { Seeing } \\
\left({ }^{\prime \prime}\right)\end{array}$ & $\begin{array}{c}\text { Moon } \\
\text { illumination }\end{array}$ & $\begin{array}{c}\text { Background noise } \\
\text { RMS (counts) }\end{array}$ \\
\hline FRB180924 & $2019-05-3115: 50: 34$ & SSO & 2.0 & $15 \%$ & 4.0 \\
& $2020-06-2917: 28: 15$ & SSO & 1.6 & $53 \%$ & 3.9 \\
\hline FRB181112 & $2019-05-3119: 27: 09$ & SSO & 1.5 & $15 \%$ & 4.5 \\
& $2020-06-2917: 39: 45$ & SSO & 3.5 & $53 \%$ & 4.4 \\
\hline FRB190102 & $2019-05-3117: 32: 31$ & SSO & 2.3 & $15 \%$ & 4.4 \\
& $2020-06-2917: 44: 52$ & SSO & 2.0 & $53 \%$ & 4.3 \\
\hline FRB190608 & $2019-06-0903: 41: 26$ & SAAO & 2.2 & $35 \%$ & 4.6 \\
& $2019-08-0814: 54: 55$ & SSO & 2.2 & $48 \%$ & 4.8 \\
& $2020-06-2917: 23: 27$ & SSO & 3.3 & $53 \%$ & 4.7 \\
\hline FRB190611 & $2019-06-1117: 31: 37$ & SSO & 1.9 & $54 \%$ & 4.6 \\
& $2019-08-0818: 01: 28$ & SSO & 2.4 & $48 \%$ & 4.7 \\
& $2020-06-2917: 55: 35$ & SSO & 3.4 & $53 \%$ & 4.9 \\
\hline FRB190711 & $2019-07-1103: 45: 05$ & SAAO & 2.3 & $66 \%$ & 6.7 \\
& $2019-08-0903: 46: 15$ & CTIO & 2.3 & $54 \%$ & 4.1 \\
\hline FRB190714 & $2020-06-2917: 12: 12$ & SSO & 1.8 & $53 \%$ & 8.8 \\
& $2019-07-1417: 03: 40$ & SAAO & 1.7 & $91 \%$ & 13.1 \\
& $2019-08-0823: 10: 34$ & CTIO & 3.5 & $54 \%$ & 4.2 \\
\hline FRB191001 & $2020-06-3008: 11: 14$ & SSO & 1.6 & $53 \%$ & 4.9 \\
& $2019-10-0520: 17: 22$ & SAAO & 2.1 & $42 \%$ & 3.9 \\
\hline & $2019-11-2600: 53: 07$ & CTIO & 2.3 & $0 \%$ & \\
\hline
\end{tabular}

Notes. ${ }^{(a)}$ SSO: Siding Spring Observatory. SAAO: South African Astronomical Observatory. CTIO: Cerro Tololo Inter-American Observatory.

few sources it has been possible to identify their host galaxies and redshifts, which confirms that they come from extragalactic sources. Most of these localizations, including those involved in this paper, have been detected by the Australian Square Kilometre Array Pathfinder (ASKAP) telescope (Johnston et al. 2008).

The first FRB was discovered in 2007 by Lorimer et al. (2007), and since then identifying the physical phenomenon (or phenomena) that gives rise to these bursts has been pursued. Many theories have been proposed for possible progenitors, including some kinds of supernovae ( $\mathrm{SNe}$; see below), compact-object mergers involving neutron stars (NSs), white dwarfs (WDs), and/or black holes (BHs), among many others (see, e.g., Platts et al. 2019, Chatterjee 2020, for compilations). The recent detection of an intense radio burst within the Milky Way from the magnetar SGR 1935+2154 hints that at least part of the FRB population originates from magnetars (CHIME/FRB Collaboration et al. 2020, Bochenek et al. 2020, Margalit et al. 2020).

Regarding SNe, some scenarios involve core-collapse supernovae (CCSNe), super-luminous supernovae (SLSNe) associated with long gamma-ray bursts (LGRBs), and Type Ia supernovae (SNe Ia; Kashiyama et al. 2013; Connor et al. 2016; Metzger et al. 2017). Young magnetars or pulsars immersed in SN remnants (see, e.g., Michilli et al. 2018) can explain the observed DMs and rotation measures (RMs). There is also the possibility that FRBs originate from WD mergers (Kashiyama et al.2013), which would produce an SN Ia. However, these models predict that it would take tens to hundreds of years for the SN ejecta to dissipate enough for FRB pulses to penetrate it (e.g., Piro 2016).

To further examine the origin of FRBs, counterparts at different wavelengths have been sought, for example in the optical range (Hardy et al. 2017; Tominaga et al. 2018; Marnoch et al. 2020; Kilpatrick et al. 2021), X-rays (Petroff et al. 2015; Scholz et al. 2016, Pilia et al. 2020, Tavani et al. 2021; Scholz et al. 2020), and gamma rays (Yamasaki et al. 2016; Cunningham et al. 2019, Guidorzi et al. 2019, 2020a). However, most of these searches have been reactive; that is, first the FRB is detected in the radio and then observations are triggered at the different wavelengths (Sun et al. 2021). This leads to a considerable time delay for these multiwavelength follow-up observations.

In Marnoch et al. (2020) the first three FRBs well localized by ASKAP were used to search for SN-like transient optical counterparts with the Very Large Telescope (VLT). They triggered one image 10 - 46 days after the burst detection and one image 233-333 days later to serve as a template for image differencing. They found no optical counterpart, so they put limits on the brightness of potential transients. They modeled light curves of different types of SNe, concluding that SNe Ia and Type IIn supernovae (IIn $\mathrm{SNe}$ ) are unlikely to be associated with any nonrepeating FRBs.

Recently, Kilpatrick et al. (2021) performed optical followup of FRB180916 on 30 second timescales (with a time delay of seconds to minutes) with the Apache Point Observatory (APO) to constrain the presence of optical emission contemporaneous with a radio burst. The repeating FRB180916 has a well-established period of $\sim 16.3$ days, which has made a coordination of observations with the Canadian Hydrogen Intensity Mapping Experiment (CHIME) possible in the radio. While CHIME did detect a radio pulse, no optical transient was apparent within a few seconds of the FRB arrival to a depth of $r \approx 24.5 \mathrm{mag}$. From these limits, Kilpatrick et al. (2021) ruled out a synchrotron maser from repeating magnetar flares where the burst energy was $>10^{44} \mathrm{erg}$ and the circumburst density was $>10^{4} \mathrm{~cm}^{-3}$.

In this work we present a search for optical transients using the Las Cumbres Observatory Global Telescope (LCOGT) network (Brown et al. 2013) at the positions of eight well-localized FRBs detected by ASKAP (Bhandari et al. 2020, Heintz et al. 2020), with data from the day of arrival of the FRB and up to $\sim 1-2$ years later. We identify no transients in our search and 
Table 2. FRB fields and epochs of observations.

\begin{tabular}{cccccccc}
\hline \hline FRB & $\begin{array}{c}\text { Arrival Time } \\
\text { (UTC) }\end{array}$ & $\begin{array}{c}1^{\text {st }} \text { epoch } \\
\text { (days) }\end{array}$ & $\begin{array}{c}2^{\text {nd }} \text { epoch } \\
\text { (days) }\end{array}$ & $\begin{array}{c}3^{\text {rd }} \text { epoch } \\
\text { (days) }\end{array}$ & $z$ & $\begin{array}{c}\text { Distance } \\
\text { Modulus }^{a}\end{array}$ & $\begin{array}{c}\text { Galactic } \\
\text { Extinction }^{b}\end{array}$ \\
\hline FRB180924 & $2018-09-2416: 23: 12$ & 249 & 644 & - & 0.3212 & 41.15 & 0.04 \\
FRB181112 & $2018-11-1217: 31: 15$ & 200 & 595 & - & 0.4755 & 42.16 & 0.05 \\
FRB190102 & $2019-01-02$ 05:38:43 & 149 & 545 & - & 0.2912 & 40.91 & 0.52 \\
FRB190608 & $2019-06-08$ 22:48:13 & 0.20 & 60.7 & 387 & 0.1177 & 38.72 & 0.11 \\
FRB190611 & $2019-06-1105: 45: 43$ & 0.49 & 58.5 & 385 & 0.3778 & 41.57 & 0.52 \\
FRB190711 & $2019-07-1101: 53: 41$ & 0.08 & 29.1 & 355 & 0.5220 & 42.41 & 0.32 \\
FRB190714 & $2019-07-14$ 05:37:13 & 0.48 & 25.7 & 352 & 0.2365 & 40.39 & 0.14 \\
FRB191001 & $2019-10-0116: 55: 36$ & 4.14 & 55.3 & 272 & 0.2340 & 40.37 & 0.07 \\
\hline
\end{tabular}

Notes. ${ }^{(a)}$ Using $\Lambda$ cold dark matter cosmological parameter from Hinshaw et al. (2013). ${ }^{(b)}$ Galactic extinction, $A_{r}$, based on Schlafly \& Finkbeiner (2011).

therefore present limits on emission for classes of luminous optical transients.

Compared to previous optical follow-up work, here we analyze a sample of several FRBs rather than single events (Hardy et al. 2017; Tominaga et al. 2018; Kilpatrick et al.2021, but see Marnoch et al. 2020). In addition, we set limits on days very close to the arrival of the bursts ( $\sim 1$ day), and our limits extend to $\sim 1$ year after the FRB emission.

Despite the modest aperture of our telescope network, we were able to constrain several extreme but possible optical transient scenarios, such as SLSNe and the brightest subtypes of $\mathrm{SNe}$ Ia, supernovae associated with gamma-ray bursts (GRB$\mathrm{SNe}$ ), and tidal disruption events (TDEs; e.g., Komossa 2015), assuming that the FRB emission coincides with the time of explosion of these transients. While TDEs are disfavored as a dominant channel based on studies of FRB offset from the host galaxy centers (e.g., Bhandari et al. 2020; Heintz et al. 2020, Mannings et al. 2020), here we test this scenario based solely on their optical light curves.

Our paper is structured as follows. In Sect. 2 we present our observation strategy and the data obtained with LCOGT. In Sect. 3 we search for optical transients and place limiting magnitudes based our observations. The results are presented in Sect. 4, and the conclusions are presented in Sect. 5.

\section{Data}

\subsection{LCOGT images}

We used data from the LCOGT network (Brown et al. 2013) for this analysis. LCOGT is a network of 23 telescopes at seven sites around the world. We used the $1 \mathrm{~m}$ telescopes with Sinistro cameras, which have a field of view $(\mathrm{FoV})$ of $26.5^{\prime} \times 26.5^{\prime}$ and a pixel size of $0.389^{\prime \prime}$. Dates, sites, seeing, Moon illumination, and background noise RMS of all observations are listed in Table 1 . The images were automatically processed through the BANZAI pipeline (McCully et al. 2018) with the latest calibration frames 11

The typical limiting apparent magnitudes obtained with our LCOGT data are approximately $22 \mathrm{mag}$ in the $r$ band (see Sect. 3.3. Nevertheless, one great advantage of using LCOGT for transient follow-up is that the network is composed of telescopes all around the world, which allowed us to obtain images of each FRB field promptly ( $<1$ day for most of the fields).

\footnotetext{
1 i.e., reduction level code 91 .
}

\subsection{Observation strategy}

Our observation strategy was to manually take an initial "epoch" of optical imaging (ideally as soon as the FRB is detected) followed by an additional epoch $30-60$ days after the radio burst to search for transient emission. For each epoch we obtained a set of ten images of $60 \mathrm{~s}$ in the $r$ band.

We triggered observations from eight FRB fields, four of which were followed-up on with rapid response, which means the first epoch could be taken within the first day after the FRB detection; however, the fields FRB180924, FRB181112, and FRB190102 have first epochs taken with much longer delays ( 100 days). We still included them in the analysis as these can constrain longer timescale transients such as SLSNe. Table 2 summarizes the time delays incurred in each epoch for our different fields.

In the first epochs of FRB190608, FRB190611, FRB190711, and FRB190714, the images were taken using localizations with larger positional uncertainties $\left(\approx 20^{\prime} \times 20^{\prime}\right)$ as given by the analysis from ASKAP based on incoherent sum data (e.g., Shannon et al. 2018). For their second and third epochs, we triggered the observations centered on the much more accurate position $\left(\sim 1-2^{\prime \prime}\right)$ as given by the subsequent coherent ASKAP analysis (Bannister et al.2019).

From this data set we can make multiple comparisons between different epochs to search for putative bright optical transients. If no transient is seen, we set limiting magnitudes (upper limits in fluxes).

\section{Analysis}

\subsection{Co-addition and photometry}

We co-added the ten images from each epoch using the SWarp software (Bertin 2010), centering on the FRB coordinates in each field, with an image resampling method, LANCZOS4, and subtracting the background from individual images before resampling.

To obtain the photometry of each FRB field, we used the software Source Extraction and Photometry (SEP; Barbary 2018, Bertin \& Arnouts 1996) on the stacked images. We empirically calibrated the magnitude zero points by performing a crossmatch between the point sources in our fields and the SkyMapper Southern Survey star catalog (Onken et al.2019). After subtracting the background estimated by SEP, we calculated fluxes of all point-like sources in our FoV using a circular aperture of 8 pixel radius $\left(\sim 3^{\prime \prime}\right)$. We did not correct for our fixed aperture, which affects the estimated flux at the order of $5 \%$. We estimated the 


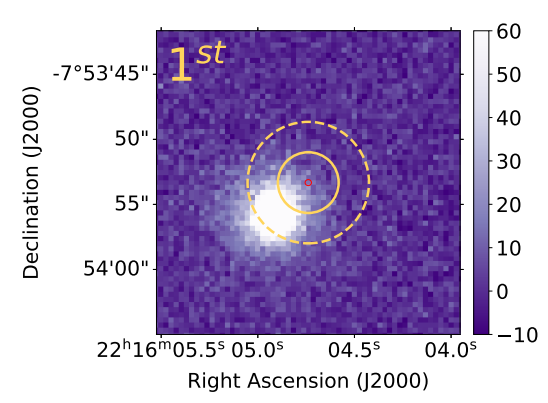

(a) FRB190608 in $1^{\text {st }}$ epoch.

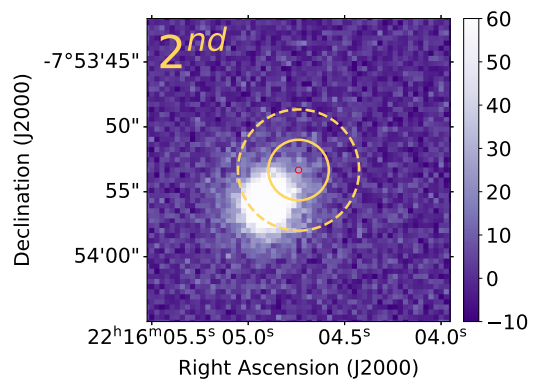

(b) FRB190608 in $2^{\text {nd }}$ epoch.

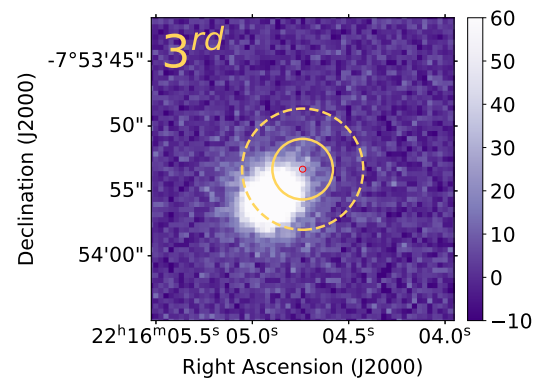

(c) FRB190608 in $3^{\text {rd }}$ epoch.
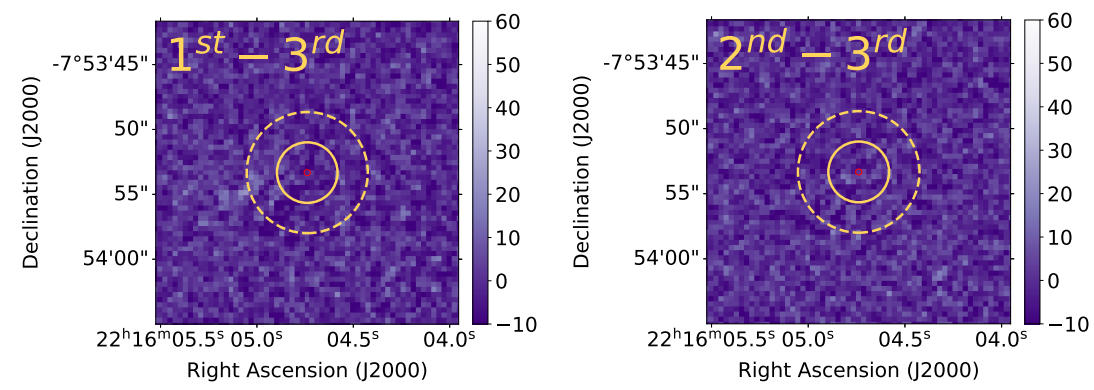

(d) Last epoch as template
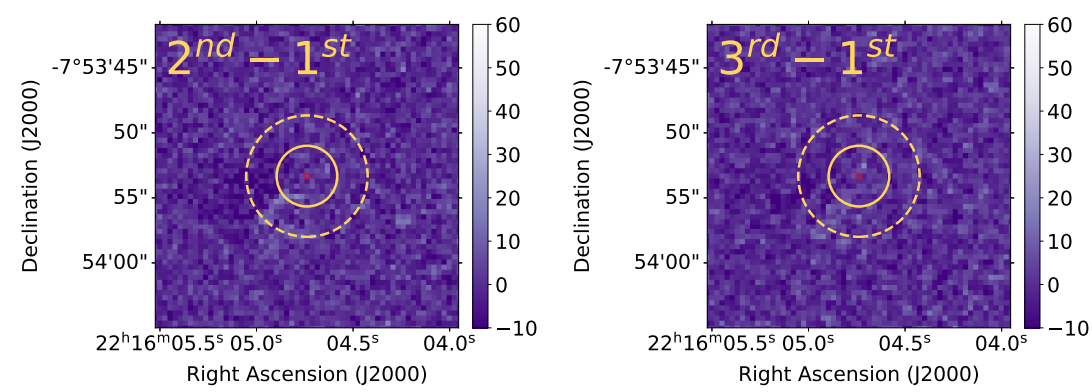

(e) First epoch as template

Fig. 1. Example of difference images for FRB190608. Panels a), b), and c) correspond to the images of each epoch of the FRB190608 observations (three epochs in total for this FRB). Panels d) and e) show the difference images using the image of the last (3) and first (1) epoch as a template, respectively. The solid and dashed circles in all the panels represent the aperture radius of one and two times the FWHM, respectively, as reference, where FWHM $=\sim 2.3^{\prime \prime}$. Our photometric analysis uses an aperture radius of two times the FWHM to set the non-detection limiting magnitudes. The red ellipse represents the uncertainty in the FRB position.

zero point for each stacked frame by crossmatching each pointlike, unsaturated source in our imaging to those in the SkyMapper catalog and within a radius of $2^{\prime \prime}$.

\subsection{Search for transients}

We searched for sources of transient optical emission at the precise FRB coordinates for each field. Images of the different epochs in a given FRB field were subtracted using the High Order Transform of PSF And Template Subtraction software (Becker 2015). The convolution parameters used are the following: The template was convolved, and we used kernel order 2, background order 1 , and ten stamps in the $x$ and $y$ dimensions.

We produced two sets of template-subtracted images. In one group, we used the image of the last epoch in a given field as the "template." In the other group, we use the image of the first epoch in a given field as the template. In both cases the template refers to the image that is subtracted from the other. Figure 1 shows an example of these subtraction groups for FRB190608.

We then ran SEP on the image difference with a circular aperture radius of twice the full width at half maximum (FWHM) and detection threshold of $1.5 \sigma$ in order to look for possible bright optical transients at the position of the eight individual FRBs. No bright optical transients were detected using this method, so we proceeded to set limiting magnitudes.

\subsection{Limiting magnitudes}

Since we did not find any optical transients in our data, we set limiting magnitudes to compare such limits with models of possible progenitors.

To estimate the limiting magnitudes, we injected artificial stars (point-like sources) of different apparent magnitudes at the positions of the FRBs in each image, in this manner mocking an unresolved optical transient. We estimated the FWHM of each LCOGT image by taking the average from three well-defined stars in the FoV (reported in the fourth column of Table 1). The artificial star is represented as a 2D Gaussian flux distribution, modeled according to the FWHM of each image.

After adding the artificial star to an individual image, we template-subtracted it (using either the first or last epoch; see Sect. 3.2. Then, we proceeded with the same automatic detec- 

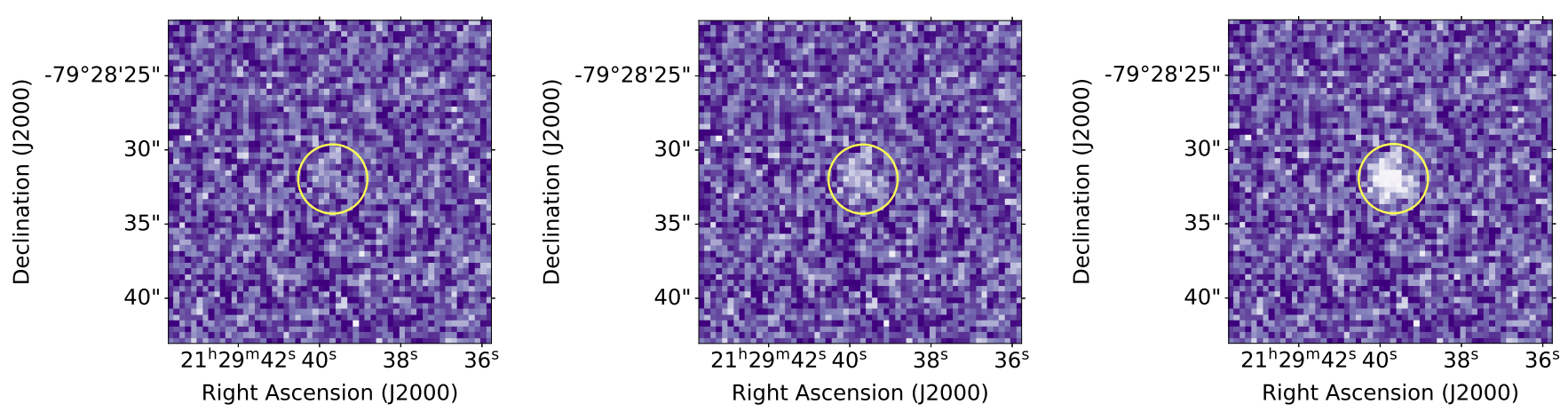

Fig. 2. Example of difference images of two epochs of the FRB190102 field, where one of them includes a mock point-like source injected with different magnitudes: a non-detected source ( $\mathrm{S} / \mathrm{N}<3$; left panel), a limiting magnitude $(\mathrm{S} / \mathrm{N}=3$; center panel), and a well-detected source ( $\mathrm{S} / \mathrm{N}$ $>3$; right panel). The pale yellow circle indicates the position where we injected the mock star with an aperture radius of the size of 1 FWHM as reference.

tion described above (see Sect. 3.2 and recorded the resulting signal-to-noise ratio $(\mathrm{S} / \mathrm{N})$ of the source recovered, calculated as the ratio between the recovered flux and its recovered flux error at the position of the mock transient (i.e., the position of the FRB). We repeated this process for artificial stars of different apparent magnitudes, ranging from 17 to $25 \mathrm{mag}$ and using $0.1 \mathrm{mag}$ steps.

When the $\mathrm{S} / \mathrm{N}$ of the recovered star was greater than 3 , we considered that a detection, and vice versa (see Fig. 2 for an illustration of this criterion). The recovered apparent magnitude was calculated through the flux, using a circular aperture radius of twice the FWHM. The limiting apparent magnitude in a given epoch was set as the fainter recovered magnitude from the difference image with $\mathrm{S} / \mathrm{N}>3$.

Since we have the redshift of the host galaxies of FRBs (i.e., the redshift of the FRB as well), we obtained the absolute magnitudes using the distance modulus and taking into account Galactic extinction (see Table 2). All the limiting magnitudes for our different epochs are presented in Table 3

\section{Results}

Figure 3 shows the limiting absolute magnitudes of the eight well-localized FRBs studied here at different epochs. The filled and empty triangles represent those that use the last and first epoch as a template, respectively (see Sect. 3.2).

Although one could expect the limits derived using the first or last epochs as templates to be the same, this is not always the case because the template subtraction process depends on the quality of both images involved in the process (e.g., background, point-spread function). Indeed, we observe a significant difference (although $\leq 1 \mathrm{mag}$ ) in the limits inferred for the second epochs of FRB190608, FRB190611, and FRB190714; these differences come mostly from the fact that the first and last epochs were observed with large differences in seeing conditions and/or moonlight illumination (see Table 1).

\subsection{Comparison of limiting magnitudes with SN light curves}

In the left panel of Fig. 3 we include different light curves of bright optical transients to compare with the limits inferred above, assuming the FRB coincides with the triggering of the transient explosion. In particular, we focus the comparison on different types of bright SNe, obtained from the Open Supernova Catalog (Guillochon et al. 2017). The catalog provides the data points of each light curve in apparent magnitudes, which we converted to absolute magnitudes by adding the distance modulus (given the redshift of the SNe provided by the catalog), taking into account Galactic extinction but not the intrinsic extinction of the host galaxy nor any $K$ correction.

For this comparison we considered SLSN, GRB-SN, and $\mathrm{SN}$ Ia types. In order to have a representative range of SN light curves, we chose the brightest and the faintest of each type available in the catalog: For SLSNe these were, respectively, SN2015bn (Nicholl et al. 2016) and SN2010md (De Cia et al. 2018); for GRB-SN they were SN1998bw (McKenzie \& Schaefer 1999) and SN2010bh (Olivares et al. 2012); and for SN Ia they were LSQ12gdj (Scalzo et al. 2014) and SN2009F (Krisciunas et al. 2017). We cut the longest sampled light curve in a given type to match the extent of the shortest. We then interpolated the corresponding data points to produce a "light-curve band" for each type, as shown in the left panel of Fig. 3. As reference, the light curve of the kilonova AT170817 (Smartt et al. 2017) connected with the gravitational wave emission GW170817 (Abbott et al. 2017) is also shown.

Assuming that the emission of the FRB coincides with the day of the explosion of the transients, we can use Fig. 3 to test possible associations. In particular, we can rule out SLSNe as a possible progenitor model for all FRBs since we have four limiting magnitudes under the light curve of the faintest SLSN cataloged. That is, if there had been an optical transient associated with an SLSN, we would have seen it in our LCOGT data at a confidence of $\sim 99.9 \%$ (see Sect. 4.2 for details). In contrast, for SNe Ia and GRB-SNe we can only rule out the bright end of the class because normal and under-luminous SNe Ia lie below our detection limits. Similarly, our data are not deep enough to rule out kilonovae as possible progenitors of FRBs.

\subsection{Quantification for ruling out SLSNe}

Thus far, we have set the detection threshold as a constant value of $1.5 \sigma$ in the difference image. This confidence interval gives us an $86.6 \%$ probability of finding the FRB between $x-1.5 \sigma<x<$ $x+1.5 \sigma$, but since they are limiting magnitudes, we integrate the probability distribution above $x+1.5 \sigma$, which results in $93.3 \%$. That is, we have a $93.3 \%$ probability that the putative optical counterpart of the FRB is below its limiting magnitude and thus a $6.7 \%$ probability that it is above the reported limiting magnitude. Because we have multiple independent limits below the faintest SLSN, we can generate more stringent limits by combining these observations. 
Table 3. Limiting magnitudes for each epoch.

\begin{tabular}{|c|c|c|c|c|c|c|c|c|}
\hline \multirow[t]{4}{*}{ FRB } & \multicolumn{8}{|c|}{ Limiting Magnitude } \\
\hline & \multicolumn{4}{|c|}{ Last epoch as template } & \multicolumn{4}{|c|}{ First epoch as template } \\
\hline & \multicolumn{2}{|c|}{$1^{\text {st }}$ epoch } & \multicolumn{2}{|c|}{$2^{\text {nd }}$ epoch } & \multicolumn{2}{|c|}{$2^{\text {nd }}$ epoch } & \multicolumn{2}{|c|}{$3^{r d}$ epoch } \\
\hline & $\begin{array}{l}\text { Apparent } \\
\text { (mag) }\end{array}$ & $\begin{array}{l}\text { Absolute } \\
\text { (mag) }\end{array}$ & $\begin{array}{l}\text { Apparent } \\
\text { (mag) }\end{array}$ & $\begin{array}{l}\text { Absolute } \\
\text { (mag) }\end{array}$ & $\begin{array}{l}\text { Apparent } \\
\text { (mag) }\end{array}$ & $\begin{array}{c}\text { Absolute } \\
\text { (mag) }\end{array}$ & $\begin{array}{l}\text { Apparent } \\
\text { (mag) }\end{array}$ & $\begin{array}{c}\text { Absolute } \\
\text { (mag) }\end{array}$ \\
\hline FRB180924 & 22.5 & -18.7 & - & - & 21.9 & -19.3 & - & - \\
\hline FRB181112 & 21.4 & -20.8 & - & - & 21.2 & -21.0 & - & - \\
\hline FRB190102 & 21.8 & -19.6 & - & - & 22.3 & -19.1 & - & - \\
\hline FRB190608 & 21.7 & -17.1 & 20.6 & -18.2 & 21.6 & -17.2 & 21.7 & -17.1 \\
\hline FRB190611 & 21.1 & -21.0 & 21.4 & -20.7 & 21.9 & -20.2 & 22.0 & -20.1 \\
\hline FRB190711 & 22.1 & -20.6 & 20.6 & -22.1 & 20.5 & -22.2 & 21.6 & -21.1 \\
\hline FRB190714 & 21.0 & -19.5 & 20.4 & -20.1 & 20.8 & -19.7 & 22.1 & -18.4 \\
\hline FRB191001 & 23.7 & -16.7 & 21.8 & -18.6 & 22.0 & -18.4 & 21.2 & -19.2 \\
\hline
\end{tabular}

Although subtracting two images from two different epochs in reverse order yields two limiting magnitudes for some epochs, it is important to note that these measurements are not independent. Thus, in order to combine limiting magnitudes, we only take the most stringent one in a given epoch for a given FRB field that lies below the faintest SLSN curve. This gives a total of four independent measurements.

Since we have four independent limiting magnitudes under the faintest SLSN light curve, the probability of being above the four limiting magnitudes is reduced to $\sim 0.002 \%$. In other words, under the assumption that all FRB emissions are simultaneous with the SLSN explosion, we can rule out SLSNe as a progenitor of FRBs with a confidence level (c.1.) of 99.998\%. On a similar basis, we can rule out the brightest subtypes of SNe Ia and GRB$\mathrm{SNe}$ at a similar confidence.

We note that these estimations do not consider systematic effects, such as different arrival times for the FRB. However, we can relax the assumption of explosion-FRB simultaneity. For this we considered time delays for the FRB that guarantee a minimum number of magnitude limits remaining under the faintest SLSN light curve (i.e., equivalent to continuously moving the SLSN light-curve band to the left in Fig. 3 and comparing it with the limits). If we take a minimum of two limiting magnitudes (corresponding to a $\sim 99.6 \%$ c.l.), we can still rule out an FRB-SLSN association for scenarios where the FRB arrival is up to 170 days after the SLSN explosion.

These estimations also do not consider systematic uncertainties in the object luminosity due to host galaxy extinction. Nevertheless, our limits do not change significantly, even with $r=1$ mag of intrinsic extinction. In such a case, we obtain three limiting magnitudes of FRBs under the SLSN light-curve band instead of four. However, if we include the limiting magnitude associated with the first epoch of FRB191001 (which is well below 1 mag of the extrapolation of the SLSN to $<1$ day earlier; see Fig. 3, our previous significance remains the same.

\subsection{Comparison of limiting magnitudes with TDE light curves}

In the right panel of Fig. 3 we have focused on the limiting magnitudes within the first few days after the arrival of the FRB. As reference, we have added light curves of two TDEs, AT2019qiz (Nicholl et al.2020) and PS1-10jh (Gezari et al.|2015), obtained from the Open TDE Catalog ${ }^{2}$

\footnotetext{
${ }^{2}$ http://TDE.space
}

In contrast to the case of SNe that have a well-defined time of explosion, for TDEs it is not possible to define an obvious initial time. For this reason, in the following we consider two broad possibilities: (1) the FRB is emitted when the TDE reaches its peak luminosity (dashed lines in the right panel of Fig. 3, and (2) the FRB is emitted 54 days or 18 days before the TDE reaches its peak luminosity (solid lines in the right panel of Fig. 3). The second possibility is motivated by being the time between the first cataloged data point and the peak luminosity of each TDE. Although arbitrary, this choice provides a range of possibilities for a putative FRB-TDE association.

Taking into account possibility (1), we have two independent limiting magnitudes under the light curve of the faintest TDE. Following the reasoning presented in Sect. 4.2, in this case we can rule out TDEs as possible progenitors of FRBs with a $\sim 99.6 \%$ c.l. Now focusing on possibility (2), we can only rule out the brightest TDE, which also has two independent limiting magnitudes under the light curve (i.e., $\sim 99.6 \%$ c.l.).

\subsection{Dearth of prompt high-energy emission from GRB-SNe}

We can put further constraints on the association of FRBs with GRB-SNe by quantifying the apparent lack of simultaneous high-energy prompt emission from the radio bursts. Since the prompt gamma-ray burst (GRB) emission is highly beamed (Gehrels et al. 2009), only a fraction of FRB-emitting sources (in the case that $\gamma$-ray emission is directly associated with them) will have a detectable precursor GRB. This can be expressed approximately as $N_{\text {tot }}=N_{\text {visible }} /\left(1-\cos \left(\theta_{\text {jet }}\right)\right)$, such that for average jet opening angles, $\theta_{\text {jet }}<10^{\circ}$ (Frail et al. 2001; Bloom et al. 2003; Guetta \& Piran 2005), only $\approx 1-2 \%$ of GRBs will be detected. Convolved with the typical fraction of GRBs detectable by, for example, Swift/BAT at any given time (Lien et al. 2014), the probability of detecting a GRB associated with an FRB is $\lesssim 1 \%$, assuming that the FRB emission is isotropic (or at least not beamed in a similar way as the GRB). We thus cannot rule out that prompt GRBs can be associated with FRBs given the still small number of well-localized FRBs (see also Palaniswamy et al. 2014; Xi et al. 2017; Martone et al. 2019, Guidorzi et al. 2020b). However, considering the wide FoV and precise timing of GRB searches, it may be feasible to use the large number of FRBs with poorer angular resolution (e.g., The CHIME/FRB Collaboration et al. 2021) to conduct a similar statistical assessment. 

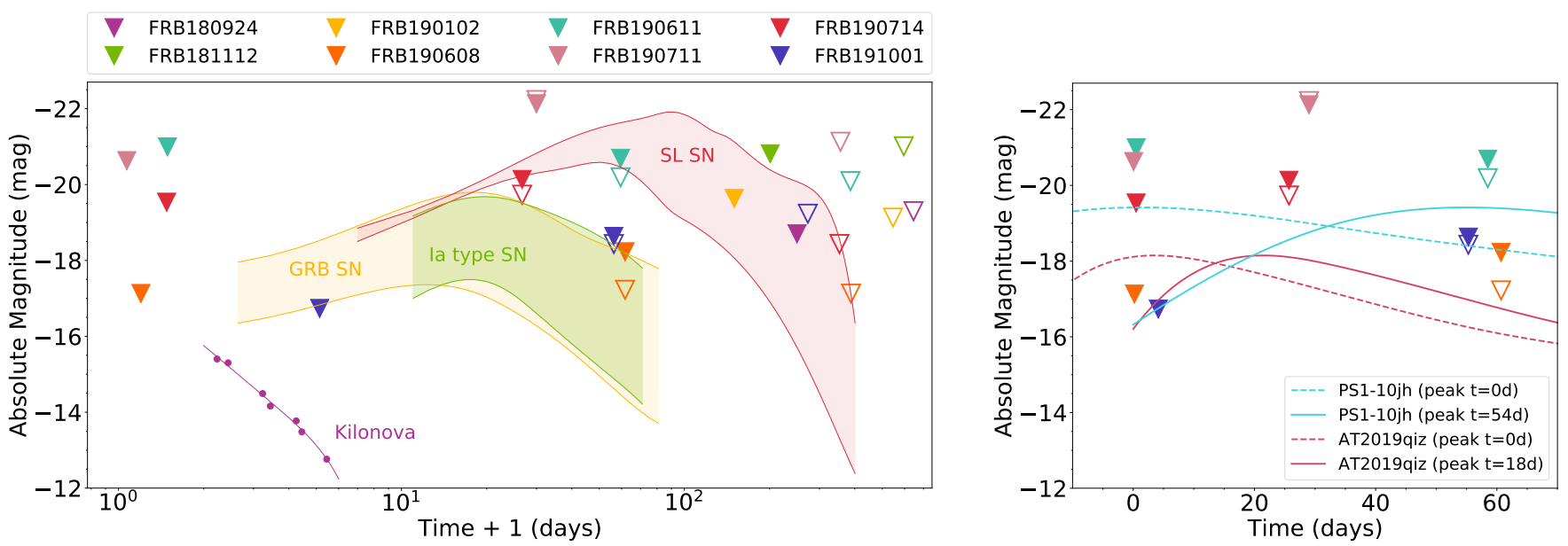

Fig. 3. Limiting absolute magnitudes at different epochs obtained for FRB positions (triangles) and light curves of different bright optical transients (colored lines and regions). Filled and open triangles correspond to the limit that uses the last and the first epoch as a template, respectively. Left panel: Light-curve bands (see Sect.4.1) for SLSNe (red), GRB-SNe (yellow), and SNe Ia (green). The light curve of the optical counterpart to a kilonova (GW170817) is also shown (purple). For the limiting magnitudes, the $x$ axis corresponds to the time since the FRB signal was received on Earth; for the light curves, the $x$ axis corresponds to the time since explosion. We emphasize that the $x$ axis is shown on a logarithmic scale $(+1$ day for convenience) and that the $y$ axis is on the astronomical magnitude scale, such that brighter objects are at the top. Right panel: Representative TDE light curves (PS1-10jh in cyan, AT2019qiz in pink) with luminosity peak times coinciding with the FRB arrival time (dashed lines) and with $t=54$ and $t=18$ days (solid lines) after the FRB arrival time. Here the $x$ axis is presented in linear scale.

\section{Conclusions}

The search for progenitors of FRBs has become a great challenge for the astronomical community over the last few years. Unlike the works carried out by Hardy et al. (2017), Tominaga et al. (2018), and Kilpatrick et al. (2021), who were looking for an optical counterpart associated only with a particular FRB, in this work we have a larger data sample, analyzing FoVs toward eight different FRBs.

Although we have not found an optical transient that solves the mystery of the progenitors of FRBs, we have ruled out SLSNe being a dominant channel, at least over the timescales probed here $(\approx 170$ days $)$ and for galaxy host extinctions of $\lesssim 1$ magnitude. Super-luminous $\mathrm{SNe}$ are rare explosions from poorly understood astrophysical phenomena associated with the ending lives of massive stars. They emit approximately 100 times more energy than typical SNe (Jerkstrand et al. 2020). With the data and the analysis obtained in this work, we can rule out the association of SLSNe with FRBs with a c.l. of $~ 99.99 \%$, assuming the FRB emission coincides with the SLSN explosion. However, this does not rule out the possibility that the FRBs come from a particular object, such as an NS, which is surrounded by an extreme environment, such as an SLSN.

For SNe Ia and GRB-SNe, we can only rule out the most luminous of each class as the faintest ones are too weak to be detected in our LCOGT data given our inferred limits and distances to the FRB hosts. Furthermore, in contrast to the work done by Marnoch et al. (2020), we have data from $\sim 1$ day after the arrival of the FRBs, which allowed us to rule out the brightest TDEs on the basis of photometry. Finally, we cannot rule out a kilonova.

Acknowledgements. We thank the anonymous referee for her/his constructive comments and suggestions which have improved the quality of this manuscript. This work makes use of observations from the Las Cumbres Observatory global telescope network (LCOGT), obtained as part of programs CN2019A-39/CLN2019A-002, CN2019B-93/CLN2019B-001 and CN2020A82/CLN2020A-001. C.N. and N.T. acknowledge support by FONDECYT grant 11191217. G.P. acknowledges support by ANID - Millennium Science Initiative - ICN12_009. K.E.H. acknowledges support by a Postdoctoral Fellowship
Grant (217690-051) from The Icelandic Research Fund. A.T.D. is the recipient of an ARC Future Fellowship (FT150100415). R.M.S. acknowledges support through ARC Future Fellowship FT190100155. L.M. acknowledges the receipt of an MQRES scholarship from Macquarie University. The Fast and Fortunate for FRB Follow-up team acknowledges support from NSF grants AST-1911140 and AST-1910471.

\section{References}

Abbott, B. P., Abbott, R., Abbott, T. D., et al. 2017, Phys. Rev. Lett., 118, 221101 Bannister, K. W., Deller, A. T., Phillips, C., et al. 2019, Science, 365, 565 Barbary, K. 2018, SEP: Source Extraction and Photometry

Becker, A. 2015, HOTPANTS: High Order Transform of PSF ANd Template Subtraction

Bertin, E. 2010, SWarp: Resampling and Co-adding FITS Images Together Bertin, E. \& Arnouts, S. 1996, A\&AS, 117, 393

Bhandari, S., Keane, E. F., Barr, E. D., et al. 2018, MNRAS, 475, 1427

Bhandari, S., Sadler, E. M., Prochaska, J. X., et al. 2020, ApJ, 895, L37 Bloom, J. S., Frail, D. A., \& Kulkarni, S. R. 2003, ApJ, 594, 674

Bochenek, C. D., Ravi, V., Belov, K. V., et al. 2020, Nature, 587, 59

Brown, T. M., Baliber, N., Bianco, F. B., et al. 2013, PASP, 125, 1031

Caleb, M., Flynn, C., Bailes, M., et al. 2017, MNRAS, 468, 3746

Chatterjee, S. 2020, arXiv e-prints, arXiv:2012.10377

Chatterjee, S., Law, C. J., Wharton, R. S., et al. 2017, Nature, 541, 58

CHIME/FRB Collaboration, Andersen, B. C., Bandura, K. M., et al. 2020, Nature, 587,54

Connor, L., Sievers, J., \& Pen, U.-L. 2016, MNRAS, 458, L19

Connor, L., van Leeuwen, J., Oostrum, L. C., et al. 2020, MNRAS, 499, 4716

Cordes, J. M. \& Chatterjee, S. 2019, ARA\&A, 57, 417

Cunningham, V., Cenko, S. B., Burns, E., et al. 2019, ApJ, 879, 40

De Cia, A., Gal-Yam, A., Rubin, A., et al. 2018, ApJ, 860, 100

Fedorova, V. A. \& Rodin, A. E. 2019, Astronomy Reports, 63, 877

Frail, D. A., Kulkarni, S. R., Sari, R., et al. 2001, ApJ, 562, L55

Gehrels, N., Ramirez-Ruiz, E., \& Fox, D. B. 2009, ARA\&A, 47, 567

Gezari, S., Chornock, R., Lawrence, A., et al. 2015, ApJ, 815, L5

Guetta, D. \& Piran, T. 2005, A\&A, 435, 421

Guidorzi, C., Marongiu, M., Martone, R., et al. 2019, ApJ, 882, 100

Guidorzi, C., Marongiu, M., Martone, R., et al. 2020a, A\&A, 637, A69

Guidorzi, C., Marongiu, M., Martone, R., et al. 2020b, A\&A, 637, A69

Guillochon, J., Parrent, J., Kelley, L. Z., \& Margutti, R. 2017, ApJ, 835, 64

Hardy, L. K., Dhillon, V. S., Spitler, L. G., et al. 2017, MNRAS, 472, 2800

Heintz, K. E., Prochaska, J. X., Simha, S., et al. 2020, ApJ, 903, 152

Hinshaw, G., Larson, D., Komatsu, E., et al. 2013, ApJS, 208, 19

Jerkstrand, A., Maeda, K., \& Kawabata, K. S. 2020, Science, 367, 415

Johnston, S., Taylor, R., Bailes, M., et al. 2008, Experimental Astronomy, 22, 151 
Kashiyama, K., Ioka, K., \& Mészáros, P. 2013, ApJ, 776, L39

Kilpatrick, C. D., Burchett, J. N., Jones, D. O., et al. 2021, ApJ, 907, L3

Komossa, S. 2015, Journal of High Energy Astrophysics, 7, 148

Krisciunas, K., Contreras, C., Burns, C. R., et al. 2017, AJ, 154, 211

Law, C. J., Butler, B. J., Prochaska, J. X., et al. 2020, ApJ, 899, 161

Lien, A., Sakamoto, T., Gehrels, N., et al. 2014, ApJ, 783, 24

Lorimer, D. R., Bailes, M., McLaughlin, M. A., Narkevic, D. J., \& Crawford, F. 2007, Science, 318, 777

Macquart, J. P., Shannon, R. M., Bannister, K. W., et al. 2019, ApJ, 872, L19

Mannings, A. G., Fong, W.-f., Simha, S., et al. 2020, arXiv e-prints, arXiv:2012.11617

Marcote, B., Nimmo, K., Hessels, J. W. T., et al. 2020, Nature, 577, 190

Margalit, B., Beniamini, P., Sridhar, N., \& Metzger, B. D. 2020, ApJ, 899, L27

Marnoch, L., Ryder, S. D., Bannister, K. W., et al. 2020, A\&A, 639, A119

Martone, R., Guidorzi, C., Margutti, R., et al. 2019, A\&A, 631, A62

Masui, K., Lin, H.-H., Sievers, J., et al. 2015, Nature, 528, 523

McCully, C., Volgenau, N. H., Harbeck, D.-R., et al. 2018, in Society of Photo-

Optical Instrumentation Engineers (SPIE) Conference Series, Vol. 10707,

Software and Cyberinfrastructure for Astronomy V, 107070K

McKenzie, E. H. \& Schaefer, B. E. 1999, PASP, 111, 964

Metzger, B. D., Berger, E., \& Margalit, B. 2017, ApJ, 841, 14

Michilli, D., Seymour, A., Hessels, J. W. T., et al. 2018, Nature, 553, 182

Nicholl, M., Berger, E., Smartt, S. J., et al. 2016, ApJ, 826, 39

Nicholl, M., Wevers, T., Oates, S. R., et al. 2020, MNRAS, 499, 482

Olivares, F., Greiner, J., Schady, P., et al. 2012, A\&A, 539, A76

Onken, C. A., Wolf, C., Bessell, M. S., et al. 2019, PASA, 36, e033

Palaniswamy, D., Wayth, R. B., Trott, C. M., et al. 2014, ApJ, 790, 63

Petroff, E., Bailes, M., Barr, E. D., et al. 2015, MNRAS, 447, 246

Petroff, E., Barr, E. D., Jameson, A., et al. 2016, PASA, 33, e045

Pilia, M., Burgay, M., Possenti, A., et al. 2020, ApJ, 896, L40

Piro, A. L. 2016, ApJ, 824, L32

Platts, E., Weltman, A., Walters, A., et al. 2019, Phys. Rep., 821, 1

Ravi, V., Catha, M., D'Addario, L., et al. 2019, Nature, 572, 352

Scalzo, R. A., Childress, M., Tucker, B., et al. 2014, MNRAS, 445, 30

Schlafly, E. F. \& Finkbeiner, D. P. 2011, ApJ, 737, 103

Scholz, P., Cook, A., Cruces, M., et al. 2020, ApJ, 901, 165

Scholz, P., Spitler, L. G., Hessels, J. W. T., et al. 2016, ApJ, 833, 177

Shannon, R. M., Macquart, J. P., Bannister, K. W., et al. 2018, Nature, 562, 386

Smartt, S. J., Chen, T. W., Jerkstrand, A., et al. 2017, Nature, 551, 75

Spitler, L. G., Cordes, J. M., Hessels, J. W. T., et al. 2014, ApJ, 790, 101

Sun, S., Yu, W., Yu, Y., \& Mao, D. 2021, ApJ, 907, 25

Tavani, M., Casentini, C., Ursi, A., et al. 2021, Nature Astronomy, 5, 401

The CHIME/FRB Collaboration, :, Amiri, M., et al. 2021, arXiv e-prints, arXiv:2106.04352

Tominaga, N., Niino, Y., Totani, T., et al. 2018, PASJ, 70, 103

Xi, S.-Q., Tam, P.-H. T., Peng, F.-K., \& Wang, X.-Y. 2017, ApJ, 842, L8

Yamasaki, S., Totani, T., \& Kawanaka, N. 2016, MNRAS, 460, 2875 